CLINICAL STUDY

\title{
Prospective evaluation of the natural course of idiopathic subclinical hypothyroidism in childhood and adolescence
}

\author{
Malgorzata Wasniewska, Mariacarolina Salerno ${ }^{1}$, Alessandra Cassio ${ }^{2}$, Andrea Corrias ${ }^{3}$, Tommaso Aversa, \\ Giuseppina Zirilli, Donatella Capalbo ${ }^{1}$, Milva Bal ${ }^{2}$, Alessandro Mussa ${ }^{3}$ and Filippo De Luca \\ Department of Pediatrics, University of Messina, 98124 Messina, Italy, ${ }^{1}$ Department of Pediatrics, University 'Federico II', Naples, Italy, ${ }^{2}$ Department of \\ Pediatrics, Univestity of Bologna, Bologna, Italy and ${ }^{3}$ Department of Pediatrics, University of Turin, Turin, Italy
}

(Correspondence should be addressed to F De Luca; Email: wasniewska@yahoo.it)

\begin{abstract}
Objective: To prospectively evaluate the course of subclinical hypothyroidism (SH) in children and adolescents with no underlying diseases and no risk factors, which might interfere with the progression of SH.

Design: Clinical status, thyroid function, and autoimmunity were prospectively evaluated at entry and after 6,12 , and 24 months in 92 young patients (mean age 8.1 \pm 3.0 years) with idiopathic SH. Results: During the study, mean TSH levels showed a trend toward a progressive decrease while $\mathrm{FT}_{4}$ levels remained unchanged. Overall, 38 patients normalized their TSH (group A): 16 patients between 6 and 12 months, and 22 patients between 12 and 24 months. Among the remaining 54 patients, the majority maintained TSH within the baseline values (group B), whereas 11 exhibited a further increase in TSH above $10 \mathrm{mU} / \mathrm{l}$ (group C). Baseline TSH and $\mathrm{FT}_{4}$ levels were similar in the patients who normalized TSH, compared with those with persistent hyperthyrotropinemia. Even in the patients of group $\mathrm{C}$, both TSH and $\mathrm{FT}_{4}$ at entry were not different with respect to those of groups $\mathrm{A}$ and $\mathrm{B}$. No patients showed any symptoms of hypothyroidism during follow-up and no changes in both height and body mass index were observed throughout the observation period.

Conclusions: (a) The natural course of TSH values in a pediatric population with idiopathic SH is characterized by a progressive decrease over time; (b) the majority of patients ( $88 \%$ ) normalized or maintained unchanged their TSH; and (c) TSH changes were not associated with either $\mathrm{FT}_{4}$ values or clinical status or auxological parameters.
\end{abstract}

European Journal of Endocrinology $160417-421$

\section{Introduction}

Subclinical hypothyroidism (SH) is a condition of moderate thyroid failure characterized by normal circulating levels of thyroid hormones with mildly elevated TSH serum concentrations. SH is a common clinical problem in adulthood and elderly, whereas its prevalence is distinctly lower in children and adolescents (1).

The natural course of $\mathrm{SH}$ in aged patients has been reported to be characterized by frequent normalization of TSH elevation, whereas the risk of progression to overt hypothyroidism was significantly greater in the ones with high TSH concentrations at baseline (2). Data concerning the natural evolution of $\mathrm{SH}$ in childhood and adolescence are very scanty. According to one of the few available follow-up studies on juvenile SH, this may be a benign and remitting process with a very low risk of evolution toward frank hypothyroidism (3).

All the available reports on the spontaneous evolution of SH in both aged and young patients have been based, up to now, on unselected study populations including patients with either thyroid disorders or other pathological causes that are well known to be able to affect SH development and evolution (1, 2, 4-7).

In the present multicenter study, we have prospectively investigated during a 2-year follow-up the spontaneous changes in $\mathrm{TSH}$ and $\mathrm{FT}_{4}$ values in a pediatric population consisting of only patients with 'idiopathic' SH, i.e., without non-thyroidal diseases and with no previous thyroid disorders or therapies affecting thyroid gland. The aim of our study design was to prospectively evaluate for the first time the natural course of SH in children and adolescents with no underlying diseases and no risk factors that might interfere with the progression of SH.

\section{Patients and methods}

\section{Study population and design}

Ninety-two selected patients (50 boys) with 'idiopathic' SH were enrolled according to well-assessed inclusion and exclusion criteria. 
All of them were younger than 15 years (mean age $8.1 \pm 3.0$ years, range 5.0-14.9) and had been referred to our pediatric endocrine clinics from the community by their pediatricians because of the incidental finding of elevated TSH concentrations in their usual annual checkup that included also TSH measurement. Out of them, 67 were prepubertal $(72.8 \%)$ and 25 were pubertal $(27.2 \%)$. All of them were of good clinical status and were not affected by any non-thyroidal illnesses. No patients exhibited either palpable goiter or symptoms generally associated with thyroid hypofunction. Thyroid ultrasonography (US) was normal and thyroid autoimmunity was negative in all the cases at the time of admission. None of them was taking iodinated drugs, lithium salts, glucocorticoids, or antiepileptic agents. Clinical history of all the patients did not evidence any antecedents of either neck radiation therapy or false positivity at congenital hypothyroidism screening. The study was performed in non-iodine-deficient areas. Therefore, in the entire study population, all the etiological causes of SH had been excluded at the time of admission.

All these patients with 'idiopathic' SH were studied as outpatients. SH was defined by elevated TSH concentrations $(5-10 \mathrm{mU} / \mathrm{l})$ in the presence of normal $\mathrm{FT}_{4}$ concentrations (10.3-24.4 pm/l). Two measurements of these hormones with an interval of 1-3 months were required to enter in the follow-up period. Patients were prospectively evaluated 6,12 , and 24 months after their admission, and at each visit clinical and hormonal (TSH and $\mathrm{FT}_{4}$ ) data and thyroid autoimmunity status were assessed. Thyroid US was reevaluated at the end of the 24-month follow-up in all the patients. In the patients who exhibited during follow-up a further increase in TSH levels above $10 \mathrm{mU} / \mathrm{l}$, at the end of follow-up, $\mathrm{L}^{-\mathrm{T}_{4}}$ treatment was begun, according to our guidelines (8) and other recommendations (9).

\section{Methods}

Thyroid function tests were determined in the fasting status and performed in the same laboratory for each subject. TSH and $\mathrm{FT}_{4}$ were determined by high specific fluorometric immunoassays. Intra- and interassay variations were less than $10 \%$.

Thyroid autoimmunity was studied by the measurement of serum levels of thyroid peroxidase antibodies (TPOAb) and the titer was considered positive for values higher than $20 \mathrm{U} / \mathrm{ml}$.

Thyroid US was performed in all subjects with a highresolution $7.5 \mathrm{MHz}$ linear transducer. The analysis of thyroid volume and echogenicity in each patient was assessed by the same operator both at entry and at the end of follow-up.

Clinical evaluation aimed to ascertain the existence of symptoms and/or signs of hypothyroidism.

Auxological assessment was based on height $(H)$ measurement and body mass index (BMI) calculation.
Standing $H$ was measured with a Harpenden stadiometer (Holtain Ltd, Crymych, Dyfed, UK). BMI was calculated as weight divided by height squared $\left(\mathrm{kg} / \mathrm{m}^{2}\right)$. To allow the comparison between different ages and genders, $H$ and BMI were expressed as s.D. scores (SDS) according to the standards assessed by Cacciari et al. (10).

\section{Statistical analysis}

Results are expressed as mean values; variability is indicated by S.D. and/or value range. For comparisons of two means, Student's $t$-test (normally distributed data) and the Mann-Whitney U-test (non-parametric data) were used. Frequency rates were compared by $\chi^{2}$ test. Correlations between quantitative variables were assessed using Pearson's correlation analysis. The level of significance was set at 0.05 .

This study design was approved by the ethical committees of our hospitals, and patients and/or their parents gave their informed consent. Appropriate consents for this study were also obtained from the Study Group for Thyroid Diseases of the Italian Society for Pediatric Endocrinology and Diabetology.

\section{Results}

\section{Changes in thyroid tests during follow-up}

Average initial TSH concentrations significantly lowered during the first 6 months of follow-up and still further during the subsequent months (Table 1). The mean decrement in TSH concentrations from baseline to the end of follow-up was $1.0 \pm 2.1 \mathrm{mU} / \mathrm{l}$. TSH decrement in the overall series was unrelated to either sex or age or pubertal status or concomitant $\mathrm{FT}_{4}$ changes.

Mean $\mathrm{FT}_{4}$ levels did not significantly change throughout the follow-up period (Table 1). In all the patients, $\mathrm{FT}_{4}$ levels remained within normal range at each time.

Thyroid US at the end of the observation period revealed significant changes compatible with Hashimoto's thyroiditis in only two patients, while in the remaining ones no significant alterations were found. TPOAb remained undetectable during the entire follow-up in all patients but two, i.e., the same two with US changes.

\section{TSH normalization}

Overall, 38 out of 92 patients normalized their TSH concentrations $(<5 \mathrm{mU} / \mathrm{l})$ during the 2-year observation period (group A): no patients during the first 6 months, 16 patients between 6 and 12 months, and 22 patients between 12 and 24 months (Table 2).

The fall in TSH values was similar in early and late normalizers.

Only two patients $(5.3 \%)$ reverted to TSH values lower than $2 \mathrm{mU} / \mathrm{l}$, while most normalizers $(68.4 \%)$ achieved TSH levels between 3 and $4.3 \mathrm{mU} / \mathrm{l}$. Among 
Table 1 Changes in average ( \pm s.D.) $\mathrm{TSH}_{\text {and }} \mathrm{FT}_{4}$ concentrations at various times during the entire follow-up in the overall study population.

\begin{tabular}{lrccr}
\hline & Baseline & $\mathbf{6}$ months & 12 months & 24 months \\
\hline $\mathrm{TSH}(\mathrm{mU} / \mathrm{l})$ & $6.1 \pm 1.3$ & $5.4 \pm 1.8^{*}$ & $5.3 \pm 2.4^{*}$ & $4.7 \pm 1.4^{\star}$ \\
$\mathrm{FT}_{4}(\mathrm{pm} / \mathrm{l})$ & $14.3 \pm 3.4$ & $14.2 \pm 3.1$ & $14.0 \pm 3.5$ & $14.0 \pm 2.5$ \\
\hline
\end{tabular}

${ }^{*} P<0.01$ versus baseline TSH.

the remaining 54 patients who did not normalize TSH concentrations during the observation period (58.7\%), the majority maintained their TSH within the baseline limits (5-10 mU/l) during the entire follow-up (group B), whereas 11 exhibited a further increase in TSH levels above $10 \mathrm{mU} / \mathrm{l}$ (between 10.5 and 15.0) despite persistently normal $\mathrm{FT}_{4}$ levels and underwent $\mathrm{L}_{-} \mathrm{T}_{4}$ therapy after the end of follow-up according to our study design (group C). In 2 out of these 11 patients, TSH increase was accompanied by both TPOAb detection and thyroid US features of Hashimoto's thyroiditis at the end of follow-up.

The percentages of patients with either decreasing or increasing or stable TSH values are scheduled in Table 2.

\section{Factors influencing TSH normalization}

Average baseline TSH concentrations $(6.0 \pm 1.5$ vs $6.2 \pm 1.1 \mathrm{mU} / \mathrm{l})$ were very similar in the patients who normalized their TSH during follow-up compared with those with persistent hyperthyrotropinemia. Even in the patients of group $\mathrm{C}$, both $\mathrm{TSH}$ and $\mathrm{FT}_{4}$ at study entry $(6.0 \pm 1.2 \mathrm{mU} / \mathrm{l}$ and $15.6 \pm 3.8 \mathrm{pm} / \mathrm{l}$ respectively) were not significantly different with respect to those of groups $\mathrm{B}(6.2 \pm 1.1$ and $14.8 \pm 3.4$ respectively) and A (6.0 \pm 1.5 and $13.6 \pm 3.4$ respectively).

Patients who normalized their TSH late (24 months) showed higher initial TSH levels $(6.2 \pm 1.7$ vs $5.8 \pm$ $1.3 \mathrm{mU} / \mathrm{l}, P<0.0125)$ and lower initial $\mathrm{FT}_{4}$ levels $(12.9 \pm 3.3$ vs $14.7 \pm 3.3 \mathrm{pm} / \mathrm{l}, P<0.05)$ with respect to those who showed early normalization (12 months). However, even the patients who were late to normalize their TSH exhibited a significant decrease in TSH values from baseline to 12 months (from $6.2 \pm 1.7$ to $5.0 \pm$ $1.6 \mathrm{mU} / \mathrm{l}, P<0.05)$, whereas no significant decrease from baseline to 12 months was observed in the patients who did not normalize their TSH during the entire follow-up.

Table 2 Percentages of patients who normalized their TSH $(<5 \mathrm{mU} / \mathrm{l})$ at various times during follow-up (group A) of the ones who maintained their TSH within the initial 5-10 mU// limits (group $\mathrm{B})$ and of those who exhibited an increase in TSH values above $10 \mathrm{mU} / \mathrm{l}$ (group C).

\begin{tabular}{lcccc}
\hline Groups (\%) & $\mathbf{6}$ months & 12 months & 24 months & Total \\
\hline A & 0 & 17.4 & 23.9 & 41.3 \\
B & 100 & 83.6 & 46.7 & 46.7 \\
C & 0 & 0 & 12.0 & 12.0 \\
\hline
\end{tabular}

The prevalence of patients who normalized their TSH during the follow-up period was significantly higher in the pubertal patients than in the prepubertal ones $(64.0$ vs $34.3 \% ; \chi^{2}=5.7, P<0.01$ ), while no differences were found between boys and girls (40.0 vs $42.8 \% ; \chi^{2}=0.1$, $P>0.05)$.

At the end of follow-up, the prevalences of pubertal patients in groups A (57.9\%) and B (23.2\%) were not significantly different when compared with the one found in group C (36.4\%).

Overall, TSH values at the time of normalization did not significantly correlate with either the corresponding values of $\mathrm{FT}_{4}$ or the time at normalization.

The prevalence of familial history of thyroid diseases was not significantly different in the patients who normalized TSH than in the ones with no TSH normalization ( 45.8 vs $54.2 \% ; \chi^{2}=1.6, P>0.05$ ).

\section{Clinical and auxological data}

None of the patients showed any clinical signs or symptoms of hypothyroidism during the entire follow-up.

During follow-up, no significant changes in the average $H$ and BMI SDS were longitudinally observed in the entire study population and in the different groups as reported in Table 3 . Both at entry and at the end of follow-up, the percentages of individuals with height deficiency were not significantly different in the entire series and in the three subgroups (Table 3).

In the overall study population, the percentage of individuals with overweight significantly decreased from entry to the end of follow-up, and this decrease was observed in both groups $\mathrm{B}\left(\chi^{2}=5.5, P<0.01\right)$ and A, although group A did not achieve a statistical relevance $\left(\chi^{2}=2.3, P>0.05\right.$; Table 3$)$. By contrast, in group $C$, the percentage of individuals with overweight remained stable from entry onward (Table 3). At the end of follow-up, the percentage of individuals with overweight was slightly higher in group $\mathrm{C}$ with respect to the one found in the remaining 81 patients (36.4 vs $16 \%$ ), but this difference did not achieve a statistical relevance $\left(\chi^{2}=2.6, P>0.05\right)$.

Data concerning the longitudinal evolution of pubertal stages, height, and BMI SDS during the entire follow-up in the patients of group $\mathrm{C}$ are analytically reported in Table 4 . An evolution of pubertal stages throughout the 2-year follow-up was found in the four patients who had already entered puberty at the beginning of the study (nos. 4, 8, 9, and 10), while the remaining seven patients remained prepubertal during the entire follow-up (Table 4). The average height SDS at the end of follow-up did not significantly differ with respect to that recorded at study entry. In the two patients who exhibited a severe height deficiency at the end of study period, height deficiency was already present at entry (nos. 4 and 8, Table 4). Even BMI SDS did not significantly change from entry 
Table 3 Average height $(H)$ and BMI SDS and percentages of individuals with either severe height deficiency $(<-2$ SDS) or overweight ( $>$ 90th percentile) at entry and at the end of follow-up in the overall study population and in the three subgroups with different TSH pattern during follow-up period.

\begin{tabular}{lcccc}
\hline & Overall $(n=92)$ & Group A $(n=38)$ & Group B $(n=43)$ & Group C $(n=11)$ \\
\hline$H$ at study entry & $-0.1 \pm 1.3$ & $0.1 \pm 1.2$ & $-0.2 \pm 1.4$ & $-0.7 \pm 1.2$ \\
$H$ at study end & $-0.1 \pm 1.3$ & $-0.1 \pm 1.2$ & $0.0 \pm 1.5$ & $-0.9 \pm 1.1$ \\
BMl at study entry & $0.6 \pm 1.7$ & $1.1 \pm 1.5$ & $0.3 \pm 1.8$ & $0.8 \pm 1.7$ \\
BMl at study end & $1.0 \pm 3.3$ & $1.8 \pm 4.4$ & $0.2 \pm 1.3$ & $0.9 \pm 1.6$ \\
Patients with H deficiency at study entry (\%) & 8.7 & 2.6 & 11.6 & 18.2 \\
Patients with H deficiency at study end (\%) & 3.3 & 0 & 2.3 & 18.2 \\
Patients with overweight at study entry (\%) & 34.8 & 36.8 & 32.6 & 36.4 \\
Patients with overweight at study end (\%) & 18.5 & 8.7 & 5.4 & 36.4 \\
\hline
\end{tabular}

onward. Three patients were obese at study entry (nos. 2, 3, and 6, Table 4), but BMI did not change during follow-up.

\section{Discussion}

To the best of our knowledge, this is the first study aiming to prospectively investigate the spontaneous evolution over time of pituitary-thyroid function in a selected series of children and adolescents with idiopathic SH. With respect to the previous studies on young (3-7) or old patients (11-14), our study design is peculiar, in that we have preliminarily excluded all the underlying diseases and the risk factors that are known to be able to affect the natural course of SH (3-7).

According to our results, the natural history of TSH serum levels in a pediatric population with idiopathic $\mathrm{SH}$ is characterized by their progressive decrease over time, with no concomitant changes in $\mathrm{FT}_{4}$ values. This 2-year prospective study shows that 38 patients (41.3\%) out of a cohort of 92 patients under age 15 years normalized their TSH values during follow-up, and that only 11 of them (12\%) underwent a deterioration of thyroid function, as demonstrated by an increase in TSH values in the range 10-15 mU/l. The risk of developing autoimmune thyroiditis in our series was unremarkable $(2.2 \%)$.

Overall, this favorable evolution was more evident in the pubertal patients than in the prepubertal ones, while it was not significantly affected by either sex, or $\mathrm{FT}_{4}$ levels or familial antecedents of thyroid diseases. In the overall study population, these changes in TSH serum concentrations over time were not associated with significant changes in clinical status and/or auxological parameters.

Our results as a whole allow us to confirm, on a larger series of patients, the conclusions by Moore (3) that juvenile SH is a benign and remitting process with a very low risk of evolution toward frank hypothyroidism. On the contrary, our results differ in some aspects from the ones previously reported in elderly patients with SH. In fact, Parle et al. investigated the evolution of 73 patients aged 60 years and found that $18 \%$ developed overt hypothyroidism and only 5\% reverted to normal TSH values, although the follow-up period was only 12 months (13). Huber $e t$ al. studied a cohort of 82 women with a mean age of 50 years over a mean observation period of 9 years and found that $28 \%$ of them developed overt hypothyroidism, but only $4 \%$ became normal (11). The greater percentage of TSH normalization found in our survey may be explained by both the absence of previous history of either thyroid or non-thyroidal diseases and the only mild $\mathrm{SH}$ of all subjects who showed TSH values ranging between 5 and $10 \mathrm{mU} / \mathrm{l}$. In fact, high baseline TSH levels, the presence of goiter, and positive thyroid autoantibodies have been demonstrated to be the main risk factors for the progression to thyroid failure $(11,13)$. It is not surprising, therefore, that a high percentage of reversion to normal TSH was also reported by Diez et al. who adopted a study design similar to the present one, by investigating only patients with mild thyroid hypofunction and no previous thyroid illnesses (2).

Table 4 Pubertal stages, height, and BMI both at entry and at the end of follow-up (lower panel) in the 11 patients of our series who exhibited TSH values $>10 \mathrm{mU} / \mathrm{l}$ during follow-up (group $\mathrm{C}$ ).

\begin{tabular}{|c|c|c|c|}
\hline Patients & $\begin{array}{l}\text { Pubertal } \\
\text { stages }\end{array}$ & Height (SDS) & BMI (SDS) \\
\hline \multicolumn{4}{|c|}{ At study entry } \\
\hline 1 & B1P1 & -0.06 & -1.2 \\
\hline 2 & G1P1 & 0.2 & 2.7 \\
\hline 3 & B1P1 & 1.3 & 2.32 \\
\hline 4 & B3P2 & -2.5 & -1.9 \\
\hline 5 & G1P1 & -1.8 & -1.07 \\
\hline 6 & G1P1 & 0.28 & 3.35 \\
\hline 7 & G1P1 & -0.08 & 0.88 \\
\hline 8 & G1P2 & -2.2 & 1.1 \\
\hline 9 & G2P2 & -1.4 & 0.9 \\
\hline 10 & G2P3 & -0.28 & 1.34 \\
\hline 11 & B1P1 & -1.0 & 0.5 \\
\hline & & $-0.7 \pm 1.2$ & $0.8 \pm 1.7$ \\
\hline \multicolumn{4}{|c|}{ At the end of follow-up } \\
\hline 1 & B1P1 & -0.5 & -1.4 \\
\hline 2 & G1P1 & -0.2 & 2.7 \\
\hline 3 & B1P1 & 1.0 & 2.3 \\
\hline 4 & B5P5 & -2.5 & -1.6 \\
\hline 5 & G1P1 & -1.9 & -0.6 \\
\hline 6 & G1P1 & 0.2 & 3.5 \\
\hline 7 & G1P1 & -0.2 & 1.3 \\
\hline 8 & G4P4 & -2.3 & 1.2 \\
\hline 9 & G5P5 & -1.4 & 1.0 \\
\hline 10 & G5P5 & -0.7 & 0.8 \\
\hline 11 & B1P1 & -1.2 & 0.5 \\
\hline \multicolumn{2}{|l|}{ Mean \pm s.D. } & $-0.9 \pm 1.1$ & $0.9 \pm 1.6$ \\
\hline
\end{tabular}


Nevertheless, it is to be underlined that in our series only a minority of patients (5.3\%) reverted to TSH values lower than $2 \mathrm{mU} / \mathrm{l}$, whereas most normalizers (68.4\%) achieved TSH levels between 3 and $4.3 \mathrm{mU} / \mathrm{l}$, a result very similar to the one recently reported by Diez et al. (12). The short duration of follow-up in the present study, however, does not allow us to predict the long-term evolution of these patients. In the early normalizers of the present study, TSH normalization was confirmed 12 months later, i.e., at the end of follow-up period. However, to further characterize the spontaneous evolution of SH in children, more prolonged follow-up studies are necessary.

Finally, in our series both early and late normalizers exhibited an early decrease in TSH, whereas no significant decrease from baseline to 12 months was recorded in the patients who did not normalize at all their TSH. Early or late occurrence of TSH normalization in our study population was mainly conditioned by baseline $\mathrm{TSH}$ and $\mathrm{FT}_{4}$ levels.

Among the possible factors influencing the interindividual variations in serum TSH, mutations and polymorphisms which occur in the genes encoding the proteins involved in the TSH pathway could play a significant role. In this regard, the direct involvement of TSH receptor (TSHR) gene in the serum TSH concentration has been proven in some cases of $\mathrm{SH}$, in which loss of function mutation has been identified $(15,16)$. However, a low prevalence of TSHR mutations has been recently reported in children with SH (17). In the present series, we did not examine TSHR gene because this was not the aim of our study.

In conclusion, (a) the natural course of TSH values in a pediatric population with idiopathic $\mathrm{SH}$ is characterized by a progressive decrease over time; (b) the majority of patients $(88 \%)$ normalized or maintained unchanged their TSH; (c) TSH changes were not associated with any changes in either $\mathrm{FT}_{4}$ values or clinical status or auxological parameters; and (d) TSH determination has no reason to be part of the routine checkup in children, apart from specific protocols.

\section{Declaration of interest}

The authors declare that there is no conflict of interest that could be perceived as prejudicing the impartiality of the research reported.

\section{Funding}

This research did not receive any specific grant from any funding agency in the public, commercial, or not-for-profit sector.

\section{References}

$1 \mathrm{Wu}$ T, Flowers JW, Tudiver F, Wilson JL \& Punyasavatsut N. Subclinical thyroid disorders and cognitive performance among adolescents in the United States. BMC Pediatrics 2006612.

2 Diez JJ \& Iglesias P. Spontaneous subclinical hypothyroidism in patients older than 55 years: an analysis of natural course and risk factors for the development of overt thyroid failure. Journal of Clinical Endocrinology and Metabolism 200489 4890-4897.
3 Moore DC. Natural course of subclinical hypothyroidism in children and adolescence. Archives of Pediatrics and Adolescent Medicine 1996150 293-297.

4 Calaciura F, Motta RM, Miscio G, Fichera G, Leonardi D, Carta A, Trischitta V, Tassi V, Sava L \& Vigneri R. Subclinical hypothyroidism in early childhood: a frequent outcome of transient neonatal hyperthyrotropinemia. Journal of Clinical Endocrinology and Metabolism 200287 3209-3214.

5 Radetti G, Gottardi E, Bona G, Corrias A, Salardi A \& Loche S. Study Group for Thyroid Diseases of the Italian Society for Pediatric Endocrinology and Diabetes (SIEDP/ISPED). The natural history of euthyroid Hashimoto's thyroiditis in children. Journal of Pediatrics $2006149827-832$.

6 Leonardi D, Polizzotti N, Carta A, Gelsomino R, Sava L, Vigneri R \& Calaciura F. Longitudinal study of thyroid function in children with mild hyperthyrotropinemia at neonatal screening for congenital hypothyroidism. Journal of Clinical Endocrinology and Metabolism 200693 2679-2685.

7 Kohler B, Schnabel D, Biebermann H \& Gruters A. Transient congenital hypothyroidism and hyperthyrotropinemia: normal thyroid function and physical development at the ages of 6-14 years. Journal of Clinical Endocrinology and Metabolism $1996 \mathbf{8 1}$ 1563-1567.

8 Arrigo T, Wasniewska M, Crisafulli G, Lombardo F, Messina MF, Rulli I, Salzano G, Valenzise M, Zirilli G \& De Luca F. Subclinical hypothyroidism: the state of the art. Journal of Endocrinological Investigation $20083179-84$.

9 Cooper DS. Clinical practice. Subclinical hypothyroidism. New England Journal of Medicine 2001345 260-265.

10 Cacciari E, Milani S \& Balsamo A. Directive Councils of SIEDP/ISPED for 1996-97 and 2002-03. Italian cross-sectional growth charts for height, weight and BMI (2 to $20 \mathrm{yr}$ ). Journal of Endocrinological Investigation 200629 581-593.

11 Huber G, Staub JJ, Meier C, Mitrache C, Guglielmetti M, Huber P \& Braverman LE. Prospective study of the spontaneous corse of subclinical hypothyroidism: prognostic value of thyrotropin, thyroid reserve, and thyroid antibodies. Journal of Clinical Endocrinology and Metabolism 200287 3221-3226.

12 Diez JJ, Iglesias P \& Burman KD. Spontaneous normalization of thyrotropin concentrations in patients with subclinical hypothyroidism. Journal of Clinical Endocrinology and Metabolism 200590 4124-4127.

13 Parle JV, Franklyn JA, Cross KW, Jones SC \& Sheppard MC. Prevalence and follow-up of abnormal thyrotropin (TSH) concentrations in the elderly in the United Kingdom. Clinical Endocrinology 199134 77-83.

14 Kabadi UM. 'Subclinical hypothyroidism'. Natural course of the syndrome during a prolonged follow-up study. Archives of Internal Medicine 1993153 957-961.

15 Alberti L, Proverbio MC, Costagliola S, Romoli R, Boldrighini B, Vigone MC, Weber G, Chiumello G, Beck-Peccoz P \& Persani L. Germline mutations of TSH receptor gene as cause of nonautoimmune subclinical hypothyroidism. Journal of Clinical Endocrinology and Metabolism 200287 2549-2555.

16 Tonacchera M, Perri A, De Marco G, Agretti P, Banco ME, Di Cosmo C, Grasso L, Vitti P, Chiovato L \& Pinchera A. Low prevalence of thyrotropin receptor mutations in a large series of subjects with sporadic and familial nonautoimmune subclinical hypothyroidism. Journal of Clinical Endocrinology and Metabolism 200489 5787-5793.

17 Teofoli F, Camilot M \& Tatò L. Lack of association between thyrotropin receptor gene polymorphisms and subclinical hypothyroidism in children. Journal of Endocrinological Investigation 200730 163-166.

Received 10 December 2008

Accepted 11 December 2008 